\title{
Trust and kinship: experimental evidence from rural India
}

\author{
Yashodha' ${ }^{1}$
}

Received: 21 January 2019 / Revised: 14 November 2019 / Accepted: 18 November 2019 /

Published online: 3 December 2019

(c) The Author(s) 2019

\begin{abstract}
The evidence on the welfare effects of kinship is mixed, suggesting both positive and adverse effects of kinship. This study looks into the differential effects of kinship on trusting and trustworthy behaviour by investigating the subjects' motives and drivers of differential behaviour towards kin and non-kin. We conducted an economic experiment with households of rural India. We found that kin are trusted more than non-kin and that differential trust towards kin and non-kin is mainly driven by higher other-regarding preferences towards kin rather than being due to differences in expected reciprocity between kin and non-kin. We observed a heterogeneous effect of kin on trustworthy behaviour: kin exhibit low trustworthiness when they are not close to other kin, while they exhibit higher trustworthiness when they have close kin in the network.
\end{abstract}

Keywords Investment and dictator games $\cdot$ Within-subject $\cdot$ Lab-in-field experiment $\cdot$ Informal contracts

JEL Classification C90 · C93 · D03 · D64

\section{Introduction}

Kinship is central among the social networks in developing countries where obligatory sharing norms and family interactions play a key role in everyday life (Rosenzweig 1988; Barr 2003; Etang et al. 2011). Banerjee and Duflo (2007) report that $50 \%$ of households with less than USD 2 per day in urban areas of developing countries have a small business, with family members as employees. Formal institutions and markets are absent or ill-developed in developing countries, where family and

Electronic supplementary material The online version of this article (https://doi.org/10.1007/s4088 1-019-00078-w) contains supplementary material, which is available to authorized users.

Yashodha

yashu.gowri@gmail.com

1 Department of Economics, University of Gothenburg, Gothenburg, Sweden 
kinship ties act as an alternative institution, facilitating information exchange (Nordman and Pasquier-Doumer 2015), resource sharing (Sadoulet et al. 1997), and risksharing, and as a source of informal credit (Rosenzweig 1988).

However, the welfare effects of kinship are mixed: evidence suggests that adverse effects of kinship exist as well (Peng 2004; Hoff and Sen 2005; Di Falco and Bulte 2011; Jakiela and Ozier 2015; Nordman and Pasquier-Doumer 2015). The literature suggests that the kinship network could act as a disincentive, as the successful members have to then redistribute their wealth amongst the less successful members of the network. This discourages members from dealing with kin, driving them to conceal their wealth by investing in non-sharable durables and reduced savings (Di Falco and Bulte 2013; Jakiela and Ozier 2015). Opting out from shared rights and obligatory kin norms may possibly result in high social sanctions from kin network members, which could be costly for an individual when formal institutional support is low.

The evidence on the dark side effect of kinship ties spurs discussion on whether to strengthen kinship networks to offset the failure of institutions and markets in developing countries (Hoff and Sen 2005). Given this backdrop on the nature of the kin network, it is important to understand the mechanism and the causes of the heterogeneous effects of kinship. Our study aims to analyse the effects of kinship on trust and trustworthiness and the mechanisms which drive the difference between kin and non-kin. We intend to identify the characteristics of kin networks which cause the heterogeneous impact of kinship. It is important and relevant from a policy point of view to understand the role of kinship in trust and trustworthiness. For example, if individuals trust only their own clan and own community members, expecting low reciprocity from non-kin, this may restrict their ability to collaborate and set boundaries for resource exchange and trade, thus limiting the scope for expansion and resulting in welfare loss.

Most of the studies which have measured kin networks have used survey data in which the kin network is identified by the subjects themselves. In such cases, the identification of the kin network has a measurement error as the subjects identify the network based on endogenous interaction, leading to a biased effect of kinship. ${ }^{1}$ Ideally, we need to employ a research design that identifies kin networks objectively in order to reduce measurement error. To this end, a unique administrative village household list was used to identify the kin network up to three generations in the village by using genealogy in order to lower the measurement error. We carried out an economic experiment in the rural area of Karnataka state in India by using a standard investment and dictator game to elicit trust and other-regarding behaviours, respectively. In the experiment, the subjects were matched to randomly selected kin and non-kin to elicit the behaviours, which reduced the bias associated with kin identification.

\footnotetext{
1 Vollan (2011) used an experimental approach which explored the trust difference between kin, friends, and un-related persons in Namibian villages by selecting kin and non-kin groups among the experimental participants, which restricts the number of kin in testifying to the difference. Since the subjects' participation was voluntary, kin who are close to each other might communicate the news and attend the experiment to explore, which may end up in having more representation of closest kin than distant kin.
} 
This study contributes to the literature in the following ways. First, unlike standard lab experiments, we use a non-student sample: our subjects are farmers from Indian villages who are involved in local informal agreements, where trust plays a crucial role in their success. Second, we exclusively account for the subjects' "otherregarding behaviours" to identify the mechanisms which drive the trust difference between kin and non-kin networks, which the literature has not considered before. ${ }^{2}$ Third, in addition to identifying the subjects' kin network (up to three generations), we meticulously measure kin network characteristics such as the subjects' closeness to the kin network to explain the heterogeneous effects of kinship. Finally, the experimental and non-experimental evidence on kinship effects comes mostly from Africa. The present study contributes to this literature by bringing in experimental evidence from Asian villages.

We found a strong kin effect in the trust game: subjects are more trusting towards kin than non-kin; furthermore, we observed that trust towards kin is greater the closer an individual is to his/her kin. The differential trust towards kin and non-kin is mainly driven by higher other-regarding behaviour such as altruism, fairness, and egalitarianism towards kin, while trust motivated by expected reciprocity remains the same for kin and non-kin. We observed a heterogeneous effect of kin on trustworthiness, which depends on the closeness among the members of the kin network. Kin exhibits low trustworthiness when they are not close to other kin, while they exhibit higher trustworthiness when they have close kin in the network.

\section{Experimental design}

\subsection{Setting}

The experiment was carried out in 29 selected villages in the Indian state of Karnataka, where there is a prevalence of informal sharing of groundwater to cultivate crops (more details in the supplementary material). These agreements resemble a non-enforceable contract structure, where trust plays a crucial role in sharing the resource and selecting partners for the agreement.

Our subjects included agents of groundwater contracts (sellers and buyers) and their corresponding kin and non-kin networks in the village. Agents of groundwater contracts were given the role of senders. Receiver subjects were randomly selected from a stratified sample of kin and non-kin on a household list of senders in the village (discussed in detail in the next section). A pre-experimental survey revealed that senders and receivers were about 50 years old and the majority of them were males (95\%), with an average education of 5 years, and smallholding farmers (2.5 acres). The Kruskal-Wallis test suggested no statistical difference between the household characteristics of senders and receivers (Table S1 in supplementary material).

\footnotetext{
2 Vollan (2011) has found that expectation from family members is lower, which he argued as a proxy for kin altruism.
} 


\subsection{Design}

We used the investment game (IG) developed by Berg et al. (1995) to elicit trust and trustworthiness. A standard dictator game (DG) by Forsythe et al. (1994) was used to elicit other-regarding behaviours (e.g., altruism, inequality aversion, fairness).

In the experiment, both senders and receivers were asked to identify the kin households in the village. We used village household lists, which consist of all household information, such as house number, name of the household head, and family members who are over 18 years old, including their photographs. To identify kin networks in the village, following the psychological literature, we focused on three generations of kinship ties, which contain genetically close as well as distant kin (Stewart-Williams 2007; Osiński 2009). ${ }^{3}$ Clear information was given about the type of relationships that exist within the three generations such as parents, siblings, cousins, aunts, uncles, nieces, nephews, children, and grandchildren and the subjects were asked to identify such households from the village list. Before the identification, we asked the subjects to help us write a genealogy to ensure that no kin was missed during the identification. In addition, the subjects were asked to rank the 10 households in the village that are closest to them, where the household ranked one is the closest. We defined closeness as a household (hh) to which "you feel a friendly, emotional, and reciprocal connection". It was emphasized that close households could be kin, non-kin, friends, and neighbours in the village. Photographs of all household members (above 18) in the village made it possible for the subjects who were unable to read to identify households.

\subsubsection{Senders' decisions}

First, we approached the senders and explained the purpose of contacting them (see the full instructions in the supplementary materials). We used a within-sample design in the IG and DG of the senders' decisions. Each sender was matched with kin and non-kin receivers randomly selected from identified kin and non-kin household lists. Senders were informed that they would be matched with two persons in the village: one of them would be randomly selected from the identified kin group and the other from the non-kin group; thereby, the subjects knew whether the matched partner was kin or non-kin, but did not know exactly the identity of the person. ${ }^{4}$ In total, the senders were involved in four decisions: one each with the matched persons in each game. At the end of the experiment, two randomly selected decisions were realized for payment.

The senders were endowed with INR 200 (about USD 3.30) for each decision and they were asked to make decisions on "how much he/she would like to send to the

\footnotetext{
${ }^{3}$ The studies used discontinuous relatedness factor $(r)$, where $r=0.75$ for parents or siblings; $r=0.5$ for grandparents, aunts, uncles, nieces, or nephews; and $r=0.25$ for cousins (Osiński 2009).

${ }^{4}$ Senders were informed that the receiver will know that the sender will be kin from their identified list; and similarly, for the receivers. The information is a common knowledge except that to whom they are matched in the kin and non-kin list.
} 
matched kin partner" and, similarly, for the matched "non-kin" partner. ${ }^{5}$ The senders were asked to make their decisions as a multiple of INR 20. We varied the order in which the subjects faced the decisions for IG and DG as well as the order of the decisions made for kin and non-kin partners, to control for order effects. ${ }^{6}$ We also elicited the risk preferences of senders. The details of the elicitation procedure can be found in Yashodha (2017).

\subsubsection{Receivers' decisions}

The receivers were randomly selected from senders' kin and non-kin lists. Each receiver was either kin or non-kin to a matched sender. ${ }^{7}$ Randomly matched kin and non-kin receivers from the senders' kin and non-kin lists were approached on the same day. We followed the same procedure as we used for the senders in identifying the receivers' kin and non-kin groups and close households in the village. ${ }^{8}$ Each receiver was involved in both the IG and DG with a matched kin or non-kin sender. In the IG, receivers made decisions about how much they would like to return to the matched sender and, in the DG, they were passive receivers. We used the strategy method to elicit the amount returned in the IG (Brandts and Charness 2000) before we informed them about the amount sent. For each possible amount that the matched kin or non-kin partner could send, the receivers were asked: "how much they would like to return to the matched partner" in multiples of INR 20. After each receiver's decision, the amount sent by the matched kin or non-kin sender was revealed for both the IG and DG. The pay-out was made to receivers by randomly selecting a decision between the IG and DG. Senders were revisited on the same day in order to pay out the randomly selected decision.

\section{Results}

\subsection{Mean comparisons}

The senders' choice in the IG (supplementary Table S2) indicates on average $31 \%$ (INR 60) of the endowment sent. This is lower than what is typically found in the literature (Camerer 2003; Johnson and Mislin 2011), where the average proportion sent was often around 50\%. The proportion sent to one's kin partner (38\%) is higher than that sent to non-kin (24\%), and the difference is statistically significant

\footnotetext{
5 We followed the endowment strategy of Glaeser et al. (2000) and Johansson-Stenman et al. (2009). The endowment is approximately equal to the one-day wage rate in the region. In addition, a participation fee of USD 1.40 was paid at the end of the experiment.

${ }^{6}$ Four possible orders: (a) Face IG and kin first, (b) Face IG and non-kin first, (c) Face DG and kin first, (d) Face DG and non-kin first.

7 In other words, kin and non-kin receivers are different samples.

8 We used genealogy for both senders and receivers to ensure that all the kin within the three generation were identified. This ensures that the matched sender appears in the kin receiver-identified kin list.
} 
(Mann-Whitney test; $p<0.01$ ). Interestingly, $17 \%$ of the subjects sent nothing and this share was higher for non-kin partners (22\%) than for kin (13\%); the Mann-Whitney test suggests that the difference in the proportion of senders sending nothing to kin and non-kin is significant $(p<0.01)$. The order of decisions faced by senders was varied, which allows us to explore between-sample comparisons. Comparing the proportion sent by senders who faced the kin decision first to the proportion sent by senders who faced the non-kin decision first (Bolded cells in supplementary Table S3), we found that the proportion sent to kin is significantly higher than that sent to non-kin, which also supports the finding of the within-subject design.

Examining the behaviour in the DG, on average, $25 \%(\mathrm{INR} \approx 50)$ of the endowment was sent to the receiver, which is consistent with the behaviour in the literature (Cox 2004; Engel 2011). However, the proportion sent to a kin partner (31\%) in the DG was considerably higher than what was sent to the non-kin partner (18\%), and the difference is statistically significant (Mann-Whitney test; $p$ value $<0.01$ ). The share of senders who sent nothing in the DG was higher when the partner was non-kin $(31 \%)$ than kin $(16 \%)$ and the difference in the proportion of senders who sent nothing to kin and non-kin was statistically significant (Mann-Whitney test; $p$ value $<0.01)$.

The average contingent proportion returned by kin and non-kin receivers was 41 and 39\%, respectively, which was higher than what was found by Ashraf et al. (2006) and consistent with the findings of Johansson-Stenman et al. (2009) and Etang et al. (2011). ${ }^{9}$ The proportion returned by kin was marginally higher than the amount returned by non-kin for all the contingent amounts. However, the mean difference was statistically significant in only 3 out of 10 contingent amounts (Table S4).

On average, both senders and receivers identified 8 kin households in the village, ranging from a minimum of 4 to a maximum of 15 households. The majority of the subjects identified 10 households that are close to them, who could be kin or nonkin. ${ }^{10}$ Merging the identified kin and close household lists, we observed on average at least one kin in the identified close household list in both the sender and receiver groups which range from 0 to 7 households. Thus, kinship does appear in the individual's closeness list, but perhaps less often than what one would expect. In contrast to our findings, in a psychological experiment by Rachlin and Jones (2008), the authors found that kin appears frequently in the subjects' social network and is given a higher closeness rank.

\subsection{Econometric analysis}

We next analysed the conditional effect of kinship on senders' behaviour in the IG and DG as follows:

\footnotetext{
9 Johansson-Stenman et al. (2009) and Etang et al. (2011) used a direct revelation approach.

10 All of the subjects have identified 10 close households and ranked them, except three senders, who could not identify more than four close households.
} 


$$
\text { sent }_{i}=\alpha+\beta \text { kin }_{i}+\mu Z_{i}+\varepsilon_{i},
$$

where sent denotes the amount sent by sender $i$ and $Z$ is a vector of variables measuring the individual characteristics of sender $i$. The constant $\alpha$ measures the amount sent to non-kin and coefficient $\beta$ measures the effect of kin in the IG or DG. We estimate Eq. (1) using the Tobit model with a lower and upper bound of INR 0 and 200 , respectively. The parameter estimates are presented in Table 2. Columns 1 and 2 indicate the estimates for the IG and DG, respectively. In both the IG and DG, coefficient $\beta$ is positive and significant, indicating that the amount sent to kin is significantly higher than the amount sent to non-kin. Being matched with kin increases the amount sent by INR 22 in the IG and by INR 23 in the DG. Further, we interact the kin dummy with the number of kin in the close hh list. The amount sent to kin receivers increases with increase in the number of kin in the close hh list by INR 9 in the IG and by INR 7 in the DG. This shows that senders exhibit high otherregarding preferences towards kin, which could be altruism, fairness, and egalitarian behaviour, and also senders trust kin more than non-kin. In order to test whether higher trust towards kin is motivated by higher expected reciprocity from kin or is due to high other-regarding behaviour towards kin, we stacked the data across the IG and DG and estimated as follows:

$$
\operatorname{sent}_{i j}=\alpha+\beta \operatorname{kin}_{i j}+\vartheta I G_{j}+\partial \operatorname{kin} * I G_{i j}+\mu Z_{i}+\varepsilon_{i j},
$$

where the constant $\alpha$ measures the amount sent to non-kin in DG. The coefficient $\beta$ measures the effect of kin in the DG: positive and significant $\beta$ indicates that a kin effect exists in the DG. The coefficient $\vartheta$ measures the difference in the amount sent between the IG and DG, with a positive coefficient implying that conditioning on the receiver type amount sent in the IG is higher than the amount sent in the DG, indicating that sender trust is motivated by expected reciprocity from the receiver. Finally, $\partial$ measures the effect of kin in the IG above and beyond the sender choice of the DG: a positive coefficient suggests that the differences in the amount sent to kin and non-kin in the IG are higher than the differences in the amount sent to kin and non-kin in the DG. This captures the impact of the difference in the expected reciprocity from kin and non-kin on sender trust behaviour.

The estimates of (2) are presented in column 3. As we can see, $\beta$ is positive and significant $(p<0.05)$, which again indicates the effect of kin persisting in the DG. Further, the effect of kin increases by INR 7 with the increase in the number of kin in the close hh list. The coefficient of IG $(\vartheta)$ is positive and significant, suggesting that the amount sent in the IG is higher than that sent in the DG by INR 15. We can infer that the sender's trust is motivated by the expected reciprocity of the receiver above and beyond other-regarding preferences towards receivers. Our coefficient of interest $\partial$ is positive and not significant, suggesting that the effect of kin in the IG is not statistically different from the effect of kin in the DG. In other words, the expected reciprocity from kin and non-kin has similar effects on sender trust. Trust motivated by expected reciprocity is similar for kin and non-kin; thus, any difference in sender trust towards kin and non-kin is not affected by the difference in the reciprocity from kin and non-kin. Further, in column 4, we explore the effect of kin in 


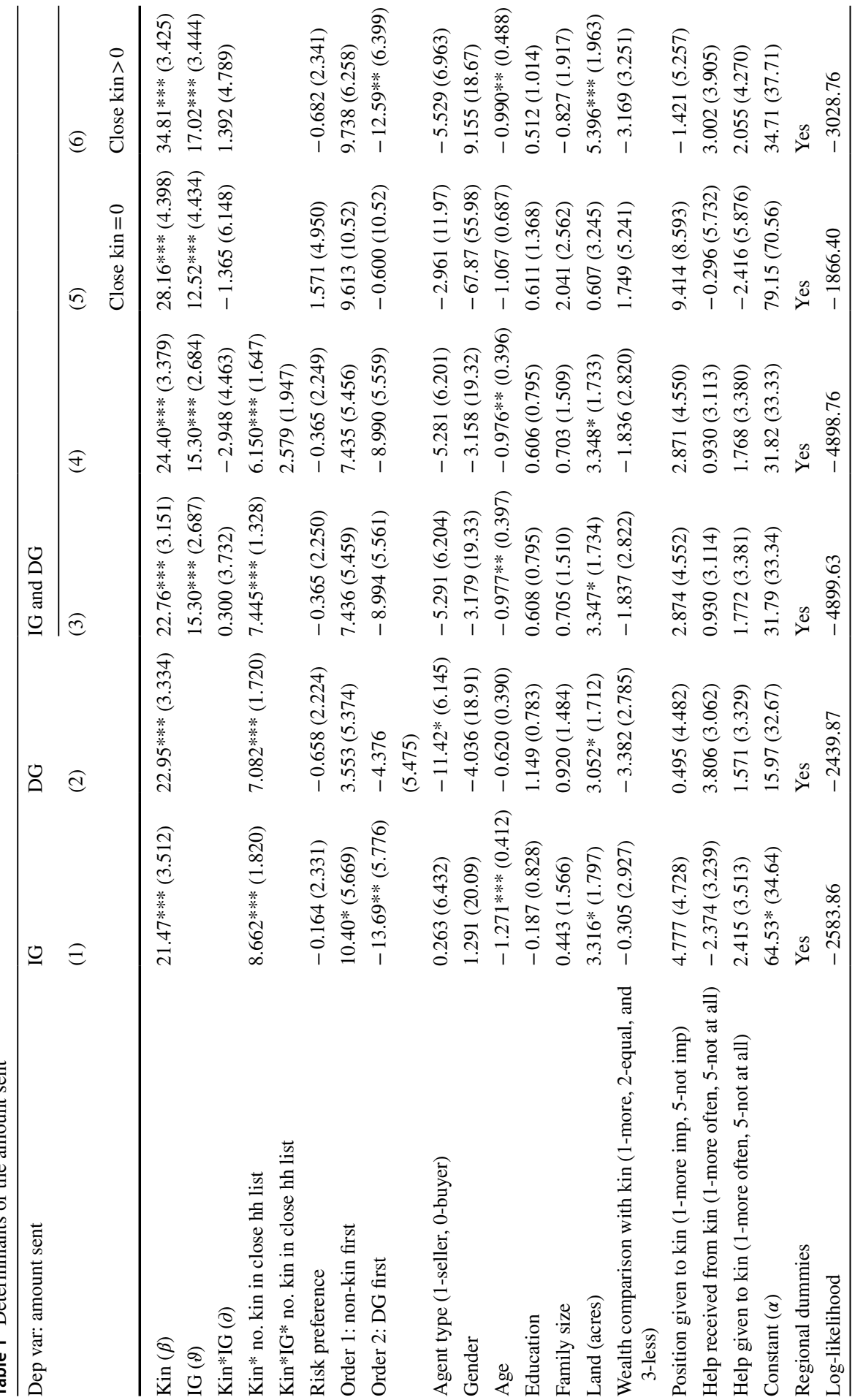




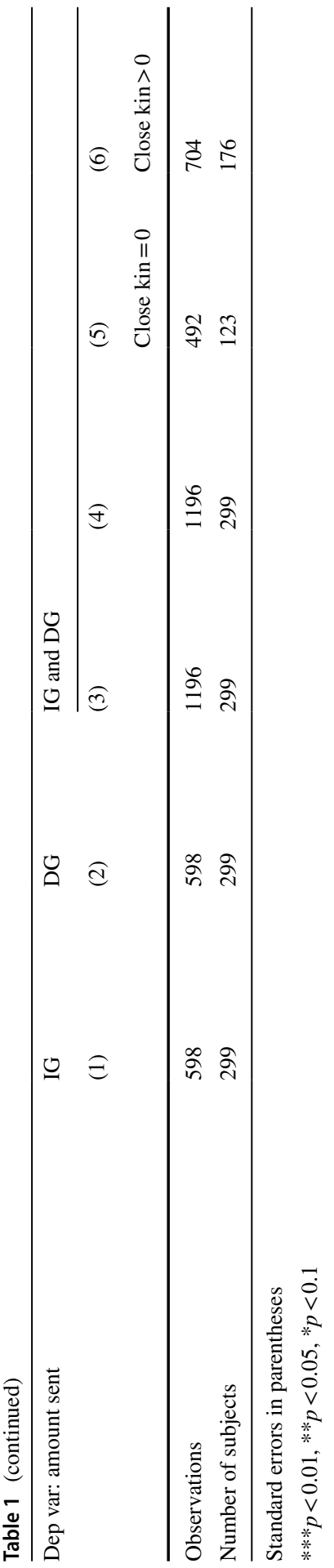


the IG by interacting the vector $\partial$ with our measure of kin closeness. As shown, the coefficient $\partial$ is now negative and is not statistically significant, which indicates that the direction of the effect of kin in the IG depends on the number of kin in the close group of households. ${ }^{11}$

We analysed the heterogeneous impact of sender closeness with kin on the effect of kin in the IG by classifying the senders into two groups based on whether the sender had kin in the close hh list. Columns 5 and 6 present estimates for the group which had no kin (not close to kin) and at least one kin in the close households list (close to kin), respectively. The magnitude of the effect of kin $(\beta)$ in the IG $(\vartheta)$ is higher for the group which had close kin than for the group which did not have kin in the close hh list. The coefficient $\partial$ is negative for senders who do not have kin in the close hh list and is positive for senders who have at least one kin in close hh list. That is, the kin effect in the IG is positive when senders are close to the kin network and the effect is negative when senders are not close to kin. However, in both groups, $\partial$ is not significantly different from zero.

We did not find a significant association between our measure of risk preference and the amount sent by senders. The order in which we present the choices affects the amount sent in the IG but not that sent in the DG. The estimates of order effects (order 1 and 2) indicate that senders send more when they face the non-kin decision first and less when they face the DG decision first. Given the general tendency of sending more to kin (positive $\beta$ ), facing the kin decision after the non-kin decision increases the total amount sent. In the second order, the subjects face decisions in the IG (which has the incentive of reciprocity from the receiver) after the DG decision (no reciprocal actions by the receiver), showing that the total amount sent in the IG decreases, which may be because the subjects learned about behaviour in the DG. ${ }^{12}$ Senders' socioeconomic characteristics such as age and land owned (a proxy for wealth) are found to have a negative and positive effect on the amount sent, respectively. Further, we control for the subjects' opinions on their wealth status in comparison with kin and also for help given to and received from kin in the village. The fitted margins for model 3 are presented in Fig. 1. Conditional on all relevant controls, the results imply that the amount sent in the IG is INR 67 and 44 for kin and non-kin, respectively, and the amount sent in the DG is INR 52 and 29 for kin and non-kin, respectively.

\footnotetext{
11 The amount sent has 11 possible discrete ordered values from INR 0, 20, $40 \ldots$ INR 200. We also estimated an ordered probit model (see supplementary Table S5) and the results found are similar in direction to those of the Tobit estimated in Table 1. For robustness, a panel fixed effect model was estimated (see Table S6) and the results are in line with the Tobit estimates in Table 1.

12 We test whether the order in which the sender faces a decision affects our coefficient of interest. We estimate (2) for the group of senders who faced a different order of decision and the results are presented in supplementary Table S7. The estimates of interest $\beta, \vartheta$, and $\partial$ for all four groups are similar in direction with the estimates of Table 1 . When senders face kin and the IG decision first, the magnitude of the effect is relatively smaller than the magnitude of other groups. This implies that senders keep a reference group when they are asked to make a series of decisions. Since senders have a tendency to send more to kin, they make kin as a reference group when they decide to send to a non-kin group. Therefore, we observe that the magnitude of the effect is higher when they face a non-kin decision first.
} 


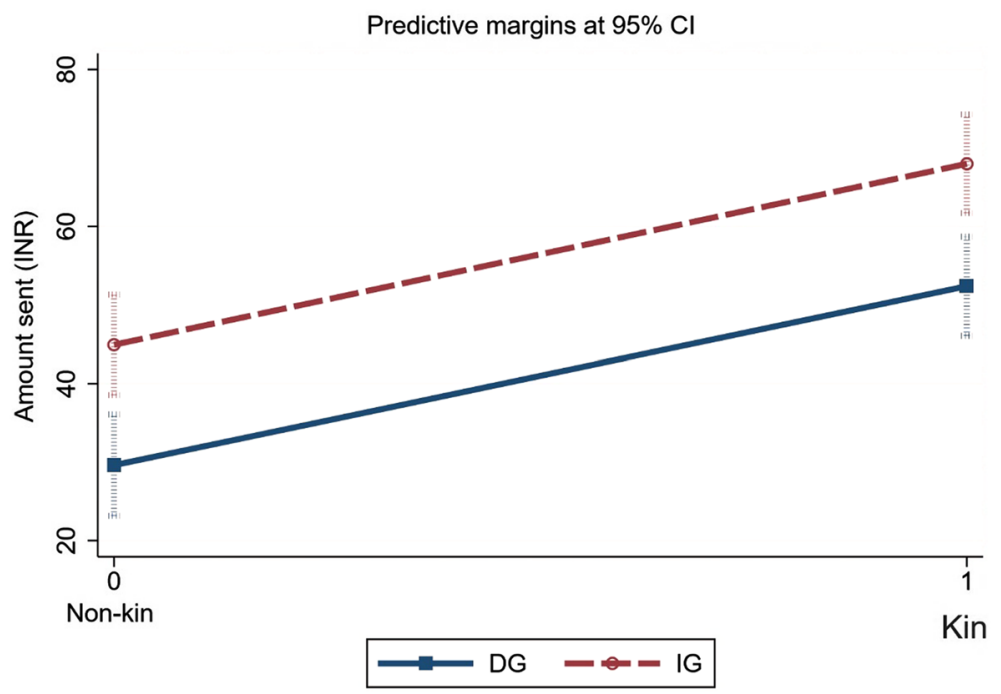

Fig. 1 Fitted margins across the IG and DG over kin and non-kin

In sum, the higher amount sent in the IG over the DG indicates that the sender's decision to trust the receiver is not only motivated by other-regarding behaviour, but is also due to expected reciprocity. We observe that the trust difference between kin and non-kin is not affected by a difference in the expected reciprocity between kin and non-kin. In addition, we show that other-regarding preferences are greater towards kin than towards non-kin. Therefore, the differences in senders trusting kin and nonkin receivers are mainly driven by the difference in other-regarding behaviour towards kin and non-kin rather than the difference in the expected reciprocity between kin and non-kin. It is noteworthy to mention that the direction of trust motivated by kin reciprocity varies depending on the sender's closeness to his/her kin network.

We analysed the determinants of the trustworthy behaviour of receivers. The results are reported in Table 2. Conditional on the contingent amount received, in column 1, we found that kin receivers return INR 13 higher than non-kin receivers, and the difference in the amount of the return is significant $(p<0.05)$. The interaction between the kin dummy and the number of kin in the close hh list changes the direction of the kin effect from positive to negative, the interaction term exhibits a positive direction, and both are statistically significant. In addition, we estimated the model, including demographic variables and other stated information about kin in the village, in column 3. The results imply that the kin effect varies depending on the kin receiver's closeness in the kin network. That is, kin receivers return an amount lower than non-kin when the receivers do not have kin in the close household list. In contrast, kin receivers return more than non-kin when they have at least one kin in the close household list. ${ }^{13}$

\footnotetext{
13 We also estimate the ordered probit model for robustness (see Table S8). The results are qualitatively similar to the estimates in Table 2.
} 
Table 2 Determinants of the amount returned

\begin{tabular}{|c|c|c|c|}
\hline \multirow[t]{2}{*}{ Variables } & \multicolumn{3}{|l|}{ Amount returned } \\
\hline & (1) & (2) & (3) \\
\hline Kin & $13.42 * *(6.650)$ & $-17.13 * *(7.787)$ & $-17.85 * *(7.714)$ \\
\hline Kin $\times$ no. kin in close hh list & & $25.61 * * *(3.754)$ & $25.66 * * *(3.693)$ \\
\hline Gender & & & $-3.385(15.91)$ \\
\hline Age & & & $-1.571 * * *(0.465)$ \\
\hline Education & & & $-1.609(1.013)$ \\
\hline Marital status & & & $-51.23(54.58)$ \\
\hline Family size & & & $2.749(2.447)$ \\
\hline Land (acres) & & & $1.641(2.000)$ \\
\hline $\begin{array}{l}\text { Wealth comparison with kin (1-more, } \\
\text { 2-equal, 3-less) }\end{array}$ & & & $-4.068(4.715)$ \\
\hline Position given kin (1-more imp, 5-not imp) & & & $-8.623 *(5.037)$ \\
\hline $\begin{array}{l}\text { Help received from kin (1-more often, 5-not } \\
\text { at all) }\end{array}$ & & & $3.109(3.836)$ \\
\hline $\begin{array}{l}\text { Help is given to kin (1-more often, 5-not at } \\
\text { all) }\end{array}$ & & & $-0.193(4.293)$ \\
\hline Constant & $1.882(4.958)$ & $2.082(4.778)$ & $155.3 * *(66.45)$ \\
\hline Fixed effect of the amount received & Yes & Yes & Yes \\
\hline Regional dummies & No & No & Yes \\
\hline Log-likelihood & $-29,089.08$ & $-29,066.70$ & $-29,054.13$ \\
\hline Number of Observations & 5980 & 5980 & 5980 \\
\hline Number of subjects & 598 & 598 & 598 \\
\hline
\end{tabular}

Standard errors in parentheses

$* * * p<0.01, * * p<0.05, * p<0.1$

Evaluating at the mean, kin return INR 8 more than non-kin receivers. In sum, the results indicate that a difference exists in the trustworthiness between kin and non-kin; however, the direction of the difference depends on the closeness to kin. Kin exhibit lower trustworthiness than non-kin when they are not close to kin and exhibit higher trustworthiness than non-kin when they are close to the kin network in the village.

\section{Discussion and conclusions}

Given the divergent arguments surrounding the welfare effect of kinship in the developing world, our study aimed to test whether individuals trust kin and consider their kin trustworthy relative to non-kin and identify the characteristics of kinship that explain the existing heterogeneous effect of kinship. Our findings confirmed that the subjects have a higher level of trust towards their kin network. This is consistent with the evidence of Vollan (2011) from African villages, where the level of trust is higher towards family members than towards other non-family groups. 
We found that senders sent higher amounts in the IG than in the DG to both kin and non-kin. Our results are consistent with Cox (2004), as senders' trust might be not only motivated by other-regarding preferences towards receivers, but might also be due to expected reciprocity from receivers. However, it is important to identify the senders' motives which contribute to the differential trust between matched agents, particularly when we investigate the trust difference towards kin and non-kin. In our study, we exclusively measured and accounted for subjects' other-regarding behaviour (through choice in the DG) to deconstruct the motivation to trust kin and non-kin recipients. Our results indicated that other-regarding preferences are higher towards kin than towards non-kin. We showed that the higher trust towards kin is mainly driven by higher other-regarding preferences towards kin rather than higher kin expected reciprocity. This result confirms the evidence of Song et al. (2012) where closer and deeper guanxi relationships in China increase the affection based trust, while not affecting the reciprocity-based trust which generates additional a social surplus in the society. Our results also provide support for the argument of Vollan (2011), where the author evidenced low level of reciprocity from family members compared with non-family members, which the author considered as a proxy for altruism, and argued that a subject's actions are motivated by kin altruism rather than by the calculated expectation of reciprocity when kin are involved. Thus, we reason that any difference in trusting kin more than non-kin in a close community such as a village is basically driven by favouring kin through other-regarding behaviours such as altruism and inequality aversion rather than a difference in the expected reciprocity from kin and non-kin. This evidence brings us to the evolutionary concept of "kin selection", in which individuals favour their kin at their own cost, which results in an inclusive fitness benefit (Hamilton 1964).

With respect to trustworthy behaviour, we found a difference between kin and non-kin receivers. The direction of the effect of kin on trustworthiness depends on the subject's closeness to the kin network. The kin effect on trustworthiness is positive when the subjects are close to the kin network and negative when they are not close to their kin network. We explain this as follows: when kin members are socially close to each other, actions are motivated not only by reciprocity but also by moral obligation, for which receivers consider a loss suffered by kin as a loss of their own. On the other hand, when subjects are socially distant within their kin network, moral obligations may fail to motivate their actions. Hence, they may not care about a loss endured by their kin when deciding on their actions.

We observed that the effect of kin is larger on trusting behaviour than on trustworthiness. Barr (2003), Song et al. (2012), and Etang et al. (2011) also found similar results, where senders' behaviour is affected by the matched partner belonging to a certain group, while the behaviour of the receiver did not change. Barr (2003) explained the findings based on the structure of the game, in which the sender (first player), before making a decision, had to think forward about another player's action. On the other hand, the receiver (second player) did not have to think forward; rather, he or she acted only in response to the actions of the sender. Under such an incentive structure, along with other-regarding behaviours such as altruism, fairness, and inequality aversion, reputation motives play a role in sender choice to send more to kin as an investment in maintaining a reputation in the kin network. Such reputational 
investments are of less relevance for receivers since they respond to the action of senders, which might cause the differential kin effect between senders and receivers.

By considering subjects' closeness to the kin network, conflicting heterogeneous effects of kinship are observed in subjects' trustworthy behaviour but not in trust behaviour. The positive kin effect supports positivist arguments (Sadoulet et al. 1997; Peng 2004), in which kinship improves the moral obligation of subjects and a negative effect of kin affirms adverse arguments on the welfare effects of kinship (Di Falco and Bulte 2011; Jakiela and Ozier 2015), for which the obligatory norms of sharing among kin breed free-riding and evasion, which hinder agents from achieving maximum welfare.

The results of the study have two implications. (1) Trusting kin more than non-kin is mainly due to higher affect-based trust toward kin rather than reciprocity-based trust, where these affections are built to individuals through norms of the society to be fair and generous towards kin. These social norms are added benefits to achieve a higher level of trust. (2) The study puts forward a new perspective on the effects of kinship by considering kin network characteristics such as kin closeness to explain the conflicting arguments found in the literature.

Acknowledgements Open access funding provided by University of Gothenburg. Financial support from the Richard C Malmsten Memorial Foundation and the Swedish International Development Cooperation Agency (SIDA) is gratefully acknowledged. I am thankful to my supervisors Fredrik Carlsson, and Håkan Eggert who provided support, insight, and expertise that greatly helped and made the study possible. I greatly acknowledge the support of Joseph Vecci for editing all the draft versions of this paper. I thank the editors and reviewers for their careful reading of my manuscript and thoughtful comments.

Open Access This article is distributed under the terms of the Creative Commons Attribution 4.0 International License (http://creativecommons.org/licenses/by/4.0/), which permits unrestricted use, distribution, and reproduction in any medium, provided you give appropriate credit to the original author(s) and the source, provide a link to the Creative Commons license, and indicate if changes were made.

\section{References}

Ashraf, N., Bohnet, I., \& Piankov, N. (2006). Decomposing trust and trustworthiness. Experimental Economics, 9, 193-208.

Banerjee, A. V., \& Duflo, E. (2007). The Economic Lives of the Poor. The Journal of Economic Perspectives, 21, 141-167.

Barr, A. (2003). Trust and expected trustworthiness: experimental evidence from Zimbabwean villages*. The Economic Journal, 113, 614-630.

Berg, J., Dickhaut, J., \& McCabe, K. (1995). Trust, reciprocity, and social history. Games and Economic Behavior, 10, 122-142.

Brandts, J., \& Charness, G. (2000). Hot vs. cold: Sequential responses and preference stability in experimental games. Experimental Economics, 2, 227-238.

Camerer, C. (2003). Behavioral game theory: Experiments in strategic interaction. Princeton: Princeton University Press.

Cox, J. C. (2004). How to identify trust and reciprocity. Games and economic behavior, 46, 260-281.

di Falco, S., \& Bulte, E. (2011). A dark side of social capital? Kinship, consumption, and savings. Journal of Development Studies, 47, 1128-1151.

di Falco, S., \& Bulte, E. (2013). The impact of kinship networks on the adoption of risk-mitigating strategies in Ethiopia. World Development, 43, 100-110.

Engel, C. (2011). Dictator games: A meta study. Experimental Economics, 14(4), 583-610. 
Etang, A., Fielding, D., \& Knowles, S. (2011). Does trust extend beyond the village? Experimental trust and social distance in Cameroon. Experimental Economics, 14, 15-35.

Forsythe, R., Horowitz, J. L., Savin, N. E., \& Sefton, M. (1994). Fairness in simple bargaining experiments. Games and Economic behavior, 6(3), 347-369.

Glaeser, E. L., Laibson, D. I., Scheinkman, J. A., \& Soutter, C. L. (2000). Measuring trust. The Quarterly Journal of Economics, 115, 811-846.

Hamilton, W. D. (1964). The genetical evolution of social behaviour. I. Journal of Theoretical Biology, 7, 1-16.

Hoff, K., \& Sen, A. (2005). The kin system as a poverty trap?. Washington, D.C.: The World Bank.

Jakiela, P. \& Ozier, O. 2015. Does Africa need a rotten kin theorem? experimental evidence from village economies. The Review of Economic Studies, rdv033.

Johansson-Stenman, O., Mahmud, M., \& Martinsson, P. (2009). Trust and religion: experimental evidence from rural Bangladesh. Economica, 76, 462-485.

Johnson, N. D., \& Mislin, A. A. (2011). Trust games: A meta-analysis. Journal of Economic Psychology, 32, 865-889.

Nordman, C. J., \& Pasquier-Doumer, L. (2015). Transitions in a West African labour market: The role of family networks. Journal of Behavioral and Experimental Economics, 54, 74-85.

Osiński, J. (2009). Kin altruism, reciprocal altruism and social discounting. Personality and Individual Differences, 47, 374-378.

Peng, Y. (2004). Kinship networks and entrepreneurs in China's transitional economy. American Journal of Sociology, 109(5), 1045-1074.

Rachlin, H., \& Jones, B. A. (2008). Altruism among relatives and non-relatives. Behavioural Processes, $79,120-123$.

Rosenzweig, M. R. (1988). Risk, implicit contracts and the family in rural areas of low-income countries. The Economic Journal, 98, 1148-1170.

Sadoulet, E., de Janvry, A., \& Fukui, S. (1997). The meaning of kinship in sharecropping contracts. American Journal of Agricultural Economics, 79, 394-406.

Song, F., Cadsby, C. B., \& Bi, Y. (2012). Trust, reciprocity, and guanxi in China: An experimental investigation. Management and Organization Review, 8(2), 397-421.

Stewart-Williams, S. (2007). Altruism among kin vs. nonkin: Effects of cost of help and reciprocal exchange. Evolution and human behavior, 28, 193-198.

Vollan, B. (2011). The difference between kinship and friendship: (Field-) experimental evidence on trust and punishment. The Journal of Socio-Economics, 40, 14-25.

Yashodha. (2017). Contract choice and trust in informal groundwater markets. https://gupea.ub.gu.se/ handle/2077/53378.

Publisher's Note Springer Nature remains neutral with regard to jurisdictional claims in published maps and institutional affiliations. 